\title{
Propagation of High Energy Electrons in Solar Plasma
}

\author{
V. Mel'nik \\ Institute of Radio Astronomy of National Academy of Sciences, 4 \\ Chervonopraporna, Kharkiv, Ukraine
}

\begin{abstract}
It is shown that high energy electrons propagate in solar plasma as beam-plasma structure, a new nonlinear object.
\end{abstract}

It was repeatedly (Magelssen, \& Smith 1977; Grognard 1985; Melrose 1990; Muschietti 1990; Benz 1993) pointed out that the self-consistent description of high energy electron propagation through plasma was the clue problem (according to Melrose (1990) "the central theoretical problem in the interpretation of nonthermal radio emission from the Sun") in the development of Type III burst theory. The difficulty of the problem is in the electron streams generate Langmuir waves that in turn influence on the electron propagation. Studying the problem over decades had not led to the commonly accepted theory.

In this paper we demonstrate that propagation of inhomogeneous beam (that is, a beam of finite length) occurs in the form of beam-plasma structure, a new nonlinear object similar to soliton.

Consider the main equations describing this object. When the quasilinear relaxation time, $\tau_{q}=\left(\omega_{p e} n^{\prime} / n\right)^{-1}\left(n^{\prime}\right.$ is the high energy electron density, $n$ is the density of background plasma, and $\omega_{p e}$ is the electron plasma frequency), is small compared with the flying-off time, $t, \tau<<t$ (namely such case is realized in solar plasma), then plateau at electron distribution function

$$
f(v, x, t)=\left\{\begin{array}{l}
p(x, t), v<u(x, t) \\
0, v>u(x, t)
\end{array}\right.
$$

and some spectral energy of Langmuir waves

$$
W(v, x, t)=\left\{\begin{array}{l}
W_{0}(v, x, t), v<u(x, t) \\
0, v>u(x, t)
\end{array}\right.
$$

are formed at every point. From the quasilinear equations of weak turbulance theory (Tsytovich 1970)

$$
\begin{gathered}
\frac{\partial f}{\partial t}+v \frac{\partial f}{\partial x}=\frac{4 \pi^{2} e^{2}}{m^{2}} \frac{\partial}{\partial v} \frac{W}{v} \frac{\partial f}{\partial v} \\
\frac{\partial W}{\partial x}=\frac{\pi \omega_{p e}}{n} v^{2} W \frac{\partial f}{\partial v}
\end{gathered}
$$

we derive the gas-dynamic equations for $p(x, t), u(x, t)$ and $W_{0}(v, x, t)$ in the standard way (Mel'nik 1995):

$$
\frac{\partial p}{\partial t}+\frac{u}{2} \frac{\partial p}{\partial x}=0, \frac{\partial u}{\partial t}+u \frac{\partial u}{\partial x}=0
$$




$$
\begin{aligned}
& \frac{\partial p}{\partial t}+v \frac{\partial p}{\partial x}=\frac{\omega_{p e}}{m} \frac{\partial}{\partial v} \frac{1}{v^{3}} \frac{\partial W_{0}}{\partial t} \\
& \frac{\partial W_{0}}{\partial t}=0, \frac{\partial u}{\partial t} W_{0}=0, v=u
\end{aligned}
$$

For the boundary and initial problems the solutions of the Equations (5-7) in the case of monoenergetic electron beam gives the nonlinear object, beam-plasma structure, (Mel'nik, Lapshin, \& Kontar 1999)

$$
\begin{gathered}
u(x, t)=\text { const }=v_{0} \\
p(x, t)=\frac{n^{\prime}}{v_{0}} \exp \left(-\frac{\left(x-v_{0} t / 2\right)^{2}}{d^{2}}\right) \\
W_{0}(v, x, t)=\frac{m}{\omega_{p e}} v^{4}\left(1-v / v_{0}\right) p(x, t)
\end{gathered}
$$

Here we present the beam-plasma structure for the initial problem when

$$
f(v, x, t=0)=n^{\prime} \exp \left(-x^{2} / d^{2}\right) \delta\left(v-v_{0}\right)
$$

In (13) $d$ is the spatial size of the initial electron cloud. It follows from Equations (8-10) that this structure consists of electrons and Langmuir waves and moves with a costant velocity. This velocity is the average velocity of electrons in the structure. Since the electron distribution function is plateau (1) at every point this velocity equals $v_{0} / 2$. The ratio of energies in electrons, $E=\int f m v^{2} / 2 d v$, and in waves, $W=\int W_{0} d k$, is 1:2 and the whole energy of beam-plasma structure, $\int(E+W) d x$, remains constant.

Numerical solutions of equations (3), (4) were obtained for different initial electron distribution functions: monoenergetic beam (Kontar, Lapshin, \& Mel'nik 1998), electron flux with $f_{i n} \sim v$ (Mel'nik, Lapshin, \& Kontar 1999), and maxwellian electron cloud $f_{\text {in }} \sim \exp \left(-v^{2} / v_{0}^{2}\right)$ (Mel'nik, Kontar, \&. Lapshinap 2000 ). These solutions show that really electrons propagate into plasma as a beam-plasma structure. The only difference is in the situation in the place of injection. In the case of monoenergetic beam some energy of initial electrons is in Langmuir waves in the injection site. If $f_{i n} \sim v$ then beam-plasma structure carries out all energy into plasma. When initial electron distribution is maxwellian the most electrons are locked in the injection site by Langmuir turbulance generated by the fastest electrons that propagate into plasma as the beam -plasma structure.

The cause of formation of beam-plasma structure is analogous that for soliton. In both cases there are two processes that compete each other. Soliton is formed by dispersion and nonlinearity. In the case of beam-plasma structure these processes are free propagation of electrons and nonlinear interaction between electrons and Langmuir waves. Propagation of "inhomogeneous" beam constantly leads out a system of electrons and Langmuir waves from the equalibrium (when $\partial f / \partial v=0$ ) state. Really, at the front of the beam faster electrons outpace slower electrons and the electron distribution with $\partial f / \partial v>0$ is formed. On the contrary at the back of the beam slower particles leg behind faster ones and this tends to restore the distribution function with $\partial f / \partial v<0$. In the first case a positive slope in $f(v)$ stimulates Langmuir waves and in the second one 
these waves are absorbed by slow electrons. All this establishes a steady state $(\partial f / \partial v=0)$. All energy of electrons converted into Langmuir waves at the beam front is absorbed by electrons at the beam back. As a result electrons accompanied by Langmuir waves propagate at large distances without any energy loss. Because the beam-plasma structure consists of Langmuir waves it can be source of emission due to plasma mechanism (Mel'nik 1999). Application of results to the theory of Type III bursts shows (Mel'nik 1991) that such properties of bursts as velocity drifts, brightness temperatures and others can be understood. 1964).

This work was partially supported by INTAS (grant No 96-0183, No 97-

\section{References}

Magelssen, G.R., \& Smith, D.F. 1977, Solar Phys., 55, 211

Grognard, R. J.-M. 1985, in Solar Radiophysics, ed.N.J.McLean \& N.R.Labrum (Cambridge: Cambridge University Press), 289

Melrose, D.B. 1990, Solar Phys., 130, 3

Muschietti, S. 1990, Solar Phys. 130, 201

Benz, A.O. 1993 Plasma Astrophysics. Kinetic process in Solar Coronae (Holland: Kluwer Academic Publishers), 299

Lamb, G.L. 1980, Elements of Soliton Theory ( New York-Chichester-BrishaneToronto: John Wiley \& Sons), 294

Tsytovich, V.N. 1970, Nonlinear Processes in a Plasma (New York: Plenum Press), 287

Mel'nik, V.N. 1995, Plasma Phys. Rep., 21, 94.

Kontar, E.P., Lapshin, V.I., \& Mel'nik, V.N. 1998, Plasma Phys. Rep., 24, 832.

Mel'nik, V.N., Lapshin, V.I., \& Kontar E.P. 1999, Solar Phys. 184, 353.

Mel'nik, V.N., 1999, in Proc. International Conf., Plasma Turbulance and Energtic Particles in Astrophysics, ed. M.Ostrovski, \& R. Schlickeiser, ( Cracow, PITS), 220

Mel'nik, V.N., Lapshin, V.I., \& Kontar E.P. 2000, Solar Phys. (to be published) Mel'nik, V.N. 1991, Kinematika i Fizika nebesnych tel, 7,59. 